\title{
QUANTITATIVE BACKSCATTERED ELECTRON ANALYSIS OF CEMENT PASTE
}

\author{
Hong Zhao' and David Darwin \\ Structural Engineering and Materials Laboratory \\ 2006 Learned Hall, University of Kansas \\ Lawrence, Kansas 66045 \\ (Refereed) \\ (Received Sept. 25, 1991)
}

\begin{abstract}
Procedures are developed to obtain reproducible quantitative data from polished specimens of cement paste using automated backscattered electron imaging. Signal production, contrast, image resolution, and imaging techniques are discussed. Preparation of a silicon-magnesium standard is described. Typical results and a statistical basis for establishing the number of frames required to provide confidence in the results are presented. The silicon-magnesium standard provides an objective method for setting both a scanning electron microscope and an image analysis system for quantitative backscattered electron analysis of phases within cement paste. The number of frames required for a selected degree of confidence decreases, but the total area required increases as magnification decreases. The number of frames is also a function of the specific phase, being greatest for unhydrated cement particles and least for inner product and calcium silicate hydrate.
\end{abstract}

\section{Introduction}

The scanning electron microscope (SEM) is a powerful tool for the study of cement-based materials. In recent years, the SEM has been employed to investigate both fractured and polished surfaces of cementitious materials, allowing individual phases within hydrated cement to be identified (1-8).

In the early work, attention was focused mainly on fractured surfaces in dried specimens (13) using secondary electron (SE) imaging. Sawed and polished surfaces were not used because polishing tended to damage the microstructure and fill in the voids with polishing debris. This problem, however, was corrected through the development of epoxy impregnation techniques $(4,9$ 11).

Polished specimens are usually investigated using backscattered electron (BSE) imaging (4). change from SE to BSE imaging is necessary because secondary electrons provide only 
TABLE 2

Mean atomic numbers and backscattering coefficients of major constituents in portland cement and their hydration products

\begin{tabular}{|c|c|c|c|}
\hline \multirow[t]{2}{*}{ Phases } & \multicolumn{2}{|c|}{ Mean Atomic Number } & \multirow{2}{*}{$\begin{array}{l}\text { Backscattering Coefficient } \\
\qquad \eta_{\operatorname{mix}}=\sum \mathrm{C}_{\mathrm{i}} \eta_{\mathrm{i}}\end{array}$} \\
\hline & $\overline{\mathrm{Z}}$ & $\overline{\mathrm{Z}}^{*}$ & \\
\hline $\mathrm{C}_{3} \mathrm{~S}$ & 15.06 & 12.67 & 0.1716 \\
\hline $\mathrm{C}_{2} \mathrm{~S}$ & 14.56 & 12.29 & 0.1662 \\
\hline $\mathrm{C}_{3} \mathrm{~A}$ & 14.34 & 12.18 & 0.1639 \\
\hline $\mathrm{C}_{4} \mathrm{AF}$ & 16.65 & 13.22 & 0.1860 \\
\hline $\mathrm{CH}$ & 14.30 & 7.60 & 0.1618 \\
\hline $\mathrm{CSH}_{1,5}$ & 12.39 & 7.10 & 0.1413 \\
\hline $\mathrm{C}_{1.5 \mathrm{SH}} \mathrm{SH}_{2}$ & 12.78 & 8.20 & 0.1455 \\
\hline $\begin{array}{l}\mathrm{C}_{3} \overline{\mathrm{AS}}_{3} \mathrm{H}_{32} \\
\text { (ettringite) }\end{array}$ & 10.76 & 5.26 & 0.1233 \\
\hline $\begin{array}{l}\mathrm{C}_{3} \mathrm{ASH}{ }_{12} \\
\text { (monosulphate) }\end{array}$ & 11.66 & 6.08 & 0.1328 \\
\hline
\end{tabular}

There are five major phases that can be identified within hydrated cement paste using BSE imaging: 1) unhydrated cement particles, $\mathrm{UH}, 2$ ) calcium hydroxide, $\mathrm{CH}, 3$ ) inner product, IP, (which generally includes the hydration products within the original boundary of the cement grain, but here more specifically refers to hydration products other than calcium hydroxide, as will be explained later), 4) calcium silicate hydrate, $\mathrm{CSH}$, and 5) voids and cracks. Based on the values shown in Table 2, it is obvious that the components of unhydrated particles have higher mean atomic numbers and backscattering coefficients than do the hydration products. Therefore, the unhydrated particles will have a higher BSE intensity than the hydration products. Of the components of unhydrated cement, $\mathrm{C}_{4} \mathrm{AF}$ has the highest mean atomic number and backscattering coefficient and, thus, $\mathrm{C}_{4} \mathrm{AF}$ will have the highest intensity in a BSE image. Calcium hydroxide, has a relatively high mean atomic number and should appear brighter than the calcium silicate hydrates, and the other hydration products. A BSE image of cement paste is presented in Fig. 1. The phases can be identified by their differences in intensity (in order of descending brightness, $\mathrm{UH}, \mathrm{CH}, \mathrm{IP}, \mathrm{CSH}$, voids and cracks).

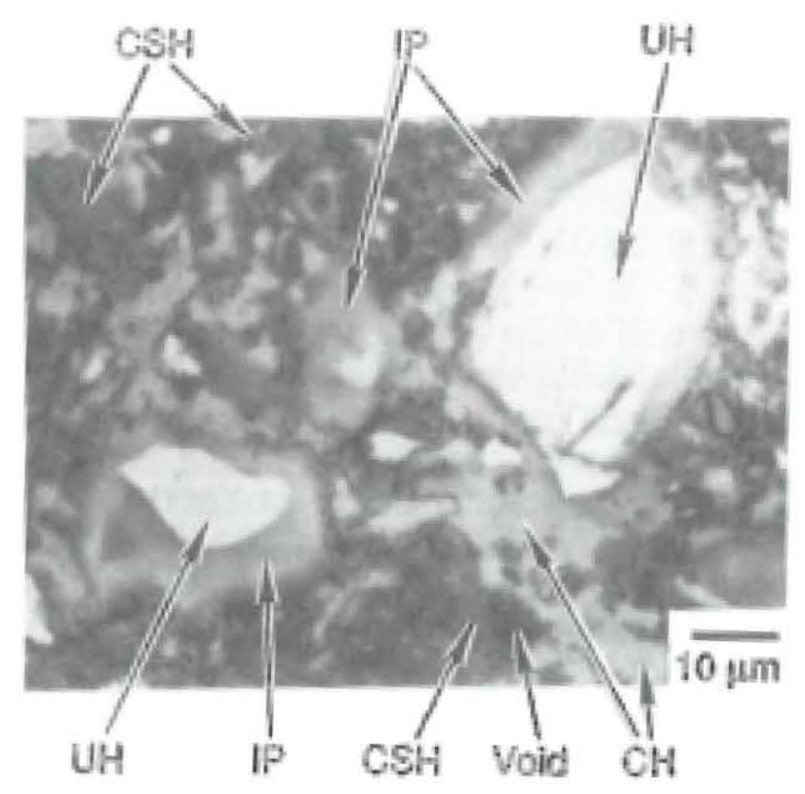

FIG. 1 BSE image of hydrated cement paste. $w / c=0.4,28$ days. 
Scrivener and Pratt (4) used similar arguments to distinguish phases within cement paste. However, they calculated the mean atomic numbers for phases within cement paste on the basis of the number of atoms instead of the weight fraction of the elemental components, resulting in incorrect predictions of the mean atomic numbers and the resulting relative intensities. The discrepancies are especially apparent for calcium hydroxide and the calcium silicate hydrates. Using their results, also shown in Table 2 , the mean atomic number for calcium hydroxide, $Z^{*}=$ 7.60, lies within the mean atomic number range for CSH, $\bar{Z}^{*}=7.10=8.20$. This, however, is inconsistent with $\mathrm{BSE}$ images in which $\mathrm{CH}$ is always brighter than $\mathrm{CSH}$. By comparing the two sets of mean atomic numbers shown in Table 2, it becomes evident that the Scrivener - Pratt values are low, especially for the hydration products.

\section{Contrast and Resolution}

In an SE or BSE image, features are visible using an SEM because the intensity of signal changes from point to point. For a BSE image, the relative difference between signals, known as the contrast, $\mathbf{C}$, can be obtained from the following equation:

$$
\mathbf{C}=\frac{\eta_{1}-\eta_{2}}{\eta_{1}}
$$

in which $\eta_{1}$ and $\eta_{2}$ are, respectively, the backscattering coefficients for high and low density materials. The smaller the value of $\mathbf{C}$, the more difficult it is to distinguish two features from each other.

A number of factors influence the contrast that can be observed using an SEM. The minimum level of contrast observable in an image is defined by the threshold equation $(16,17)$ :

$$
\mathrm{i}_{B}>\frac{4 \times 10^{-12}}{\varepsilon \mathrm{C}^{2} \mathrm{t}_{\mathrm{f}}}
$$

in which $i_{B}$ is the minimum beam current (threshold current) required to provide contrast $C$, $t_{f}$ is the time required to scan a $1000 \times 1000$ pixel frame (typical SEM pixel density for a photomicrograph), and $\varepsilon$ is the detector "efficiency" (ratio of signal current to beam current). Eq. 7 is useful in determining if specific features can be imaged with an SEM and helps point out the factors which govern the available contrast. The number in the numerator is proportional to the number of pixels in an image and will decrease for a frame with a lower pixel density. For example, the image analysis system (IAS) used in this study has a pixel density of $512 \times 480$, reducing the numerator to about $1 \times 10^{-12}$ for the same contrast available on the higher density pixel image. Thus, a frame scan time, $t_{f}$ on an LAS of $32 \mathrm{sec}$ will produce approximately the same contrast as obtained in a photomicrograph scanned for (the typical) $128 \mathrm{sec}$. The potential resolution of the $512 \times 480$ pixel image would, of course, be less than the potential resolution of the $1000 \times 1000$ pixel image.

For given values of detector efficiency, $\varepsilon$, and frame time, $t$, contrast will depend on the beam current, $i_{B}$. Beam current depends on the beam electron accelerating voltage (and thus beam electron energy, $\mathrm{E}_{\mathrm{B}}$ ) and the diameter of the electron probe, $\mathrm{d}_{\mathrm{p}}$. $\mathrm{i}_{\mathrm{B}}$ can be measured and directly controlled on an SEM. As the accelerating voltage is decreased, $\mathrm{d}_{\mathrm{p}}$ must be increased to maintain a particular beam current. Since the resolution obtained with SE's depends on probe diameter, resolution will degrade with reduced values of accelerating voltage, if $\mathrm{i}_{\mathrm{B}}$ is maintained to maintain contrast. On the other hand, since the sampling volume for BSE's depends on beam electron energy, resolution can be improved somewhat by reducing $\mathrm{E}_{\mathrm{o}}$. However, there is a strict limit, because, at low $\mathrm{E}_{\mathrm{o}}$, probe diameters can exceed the diameter of the sampling volume produced

with a high $\mathrm{E}_{\mathrm{g}}$; then the only way to improve resolution is to decrease $\mathrm{d}_{\mathrm{p}}$ (and thus $\mathrm{i}_{\mathrm{B}}$ ) with a resulting increase in $\mathrm{C}$, making it more difficult to distinguish features.

The ultimate resolution available with BSE's from a bulk sample is on the order of $0.5 \mu \mathrm{m}$ (about one-half the diameter of the sampling volume), except when adjacent materials have large differences in backscattering coefficient, $\eta$. Thus, even a sharp interface between $\mathrm{CH}$ and CSH not appear to be sharply resolved at magnifications much above $200 \mathrm{x}$, while the edge of a void 
or crack filled with epoxy can be clearly distinguished with a resolution limited only by $\mathrm{d}_{p}$ and the pixel size. The relatively poor resolution of boundaries between phases with similar mean atomic numbers makes the use of the technique questionable at magnifications much above $2000 \mathrm{x}$, except for imaging voids and cracks.

\section{Application of Image Analysis System}

An image analysis system (IAS) can be used for feature identification and quantitative analysis of images obtained from an SEM. The IAS used in this study has the ability to distinguish 256 gray levels, dark to bright, ranging from 0 to 255 . When an image is transferred from the SEM to the IAS, pixels with gray levels representing the signal intensities from corresponding points on the specimen form the image on the monitor. LAS's can divide the pixels into groups based on gray level. This is referred to as creating thresholds for gray level ranges or segmenting the image. This allows quantitative analysis to be carried out on groups of pixels with like intensities.

\section{Experimental Program}

The techniques described in this paper were developed in conjunction with a study of the structure of hydrated cement paste. The following sections emphasize those aspects of the study that relate to BSE imaging.

\section{Cement Paste}

The cement paste specimens illustrated in this paper were prepared with Type I portland cement at a water-cement ratio of 0.4 and cured at $72^{\circ} \mathrm{F}$ for 28 days in lime-saturated water.

\section{Preparation of the Standard}

Use of the SEM and IAS for reproducible quantitative analysis requires a two-material standard. The standard developed in this study consists of a thin silicon wafer $(100 \mu \mathrm{m})$ mounted

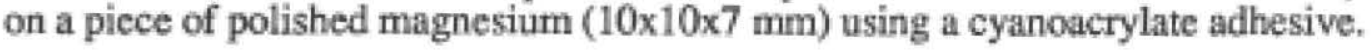

To prepare the standard, the magnesium was polished using silicon carbide paper, starting with 320 grade and proceeding to 600,1000 , and 2000 grades. Polishing with diamond paste followed, progressing from $3 \mu \mathrm{m}$ to $1 \mu \mathrm{m}$ to $0.5 \mu \mathrm{m}$ paste. The surface was cleaned after each stage of polishing with a lint free cloth and a commercial compressed gas cleaner.

The standard was mounted on an aluminum specimen stub, and colloidal graphite was used to provide electrical conductivity between the silicon, the magnesium, and the specimen stub.

Although a thinner layer of silicon would have worked as well, the $100 \mu \mathrm{m}$ wafer served the purpose quite well, since the high depth of field inherent in the SEM allows it to focus on the magnesium and silicon simultaneously. The interaction volume for silicon is fully contained within the wafer. Using an accelerating voltage of $25.0 \mathrm{kV}$, the depth of the interaction volume for silicon, $\mathrm{R}_{\mathrm{KO}}=6.85 \mu \mathrm{m}$ (Eq. 1), is far less than the $100 \mu \mathrm{m}$ thickness, insuring that beam electrons will not break through the silicon wafer and produce BSE signals with mixed information. The values of $\mathrm{Z}, \mathrm{A}$, and $\eta$ for magnesium and silicon are given in Table 1.

\section{Specimen Preparation}

In preparing for imaging in the SEM, cement paste specimens were sectioned into $1 \mathrm{~mm}$ thick segments using a high speed diamond saw. The specimens were oven dried at $105^{\circ} \mathrm{C}$ for 24 hours, cooled in a desiccator cabinet, and impregnated with an ultralow viscosity epoxy (13). A vacuum saturation technique was used for epoxy impregnation. The specimens were first subjected to a low level vacuum (50 torr.). Epoxy was drawn into the chamber under vacuuts pressure, and then normal pressure was restored. The epoxy was polymerized at $60^{\circ} \mathrm{C}$ for 24 hours.

Following polymerization, specimens were polished using progressively finer grades of silicon carbide paper and diamond paste in the same manner used for the standard. After polishing was complete, specimens were mounted on aluminum stubs and coated with a 20 nm layer of gok palladium or carbon. 


\section{Imaging Techniques}

The backscattered electron detector used in this study consisted of an array of four tilted solid state detectors. The silicon-magnesium standard, a Faraday cup, and cement paste specimens were mounted on a multiple specimen holder and placed in the SEM chamber.

Initial Settings for the SEM consisted of $25.0 \mathrm{kV}$ accelerating voltage, $100 \mathrm{~nm}$ electron probe diameter, zero degree stage tilt, and a free working distance (FWD) of $25.5 \mathrm{~mm}$. The Faraday cup was used to adjust the beam current, $\mathrm{i}_{\mathrm{B}}$, to the desired value $\left(0.2 \times 10^{-9}\right.$ to $\left.1 \times 10^{-9} \mathrm{amps}\right)$. To quantify the detector settings, a scale (ranging from +25 for full white to -25 for full black) was placed on the SEM videoscope, as shown in Fig. 2.

\section{Videoscope Screen}

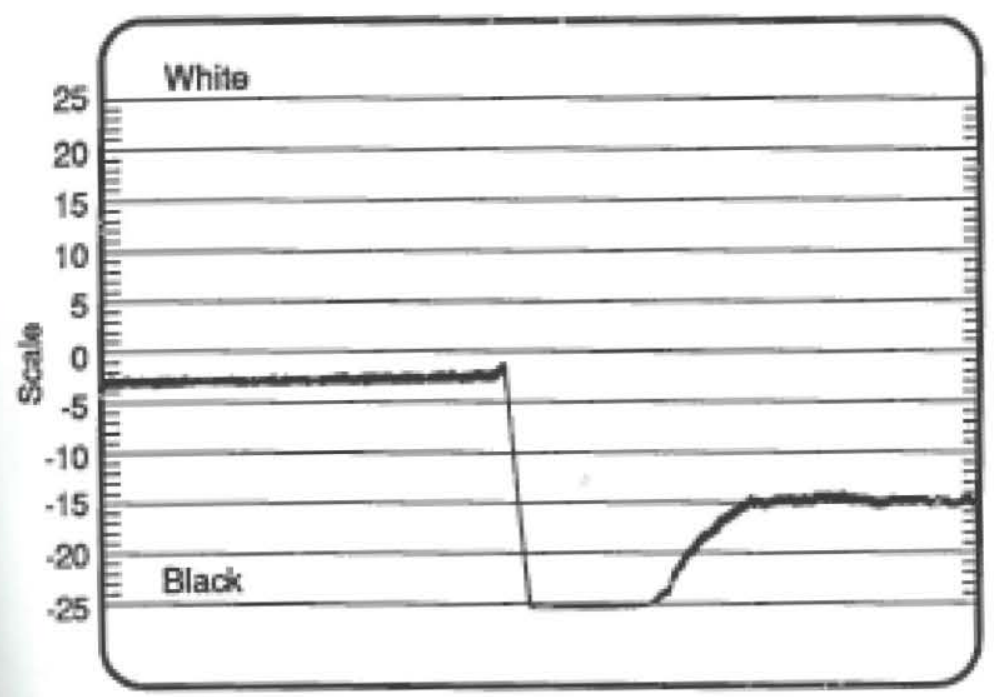

FIG. 2 Videoscope with scale and signal produced by Si-Mg standard.

Backscattered Electron Detector Adjustment was made by using the silicon/magnesium standard. Following beam current calibration, BSD adjustment started by placing the Si-Mg standard in the viewing region and bringing the interface of silicon and magnesium to the vertical center line of the CRT screen. The electron beam was focused on the edge of the silicon, and a BSE image was obtained. The line-scan mode was used to adjust the contrast and brightness settings of the BSD. A typical step function for the BSE signal of the standard on the videoscope is shown in Fig. 2. The signal at the left is silicon and the signal at the right is magnesium.

Two BSD settings were established to yield the desired information. To differentiate between the five major phases within cement paste (treating unhydrated materials as a single phase), the standard setting consisted of mean videoscope signal intensities of -3 for silicon and -15 for magnesium. To differentiate between phases within the unhydrated particles, the standard setting consisted of mean signal intensities of -8 for silicon and -18 for magnesium. The second setting provided lower contrast and brightness, which allowed the high intensity BSE images of the unhydrated materials to be distinguished from each other. The different settings are necessary, not because of limitations in the instrumentation, but because of the inability of the human eye to distinguish closely spaced gray levels (18).

Image Analysis System Calibration followed BSD calibration. Calibration of the IAS with mean SEM signal intensities of $-3(\mathrm{Si})$ and $-15(\mathrm{Mg})$ is described below. The procedure for signal intensities of $-8(\mathrm{Si})$ and $-18(\mathrm{Mg})$ is similar.

A $300 \mathrm{x}$ image of the standard was acquired using the minimum scan speed (frame time $=32$ and the maximum contrast setting on the LAS. A gray level threshold file was used to verify the system was properly calibrated. As described earlier, the LAS has the ability to distinguish gray levels ranging from 0 to 255 . An upper limit of 240 was used in the current study (this reduction was based on analysis requirements that do not impact the current paper). These tized gray levels represent the full range of signal intensities. In a threshold file, single user- 
defined colors can be assigned to selected portions of the full range. The threshold file used for the Si-Mg standard used red to represent the gray levels of Si, ranging from 102 to 138 , and blue to represent the gray levels of $\mathrm{Mg}$, ranging from 24 to 62 . Yellow and purple were used for the gray lcvels above silicon and for the gray levels between $\mathrm{Si}$ and $\mathrm{Mg}$, respectively. Black represented gray levels below 24 .

A color image of the standard was obtained by using the threshold file. With the brightness and contours on the LAS properly adjusted, the color image showed a virtually solid red section for silicon and a solid blue section for magnesium, with a black strip running along the Si-Mg interface (the vertical surface produced few BSE in the direction the detector) and some discrete spots of yellow and purple caused by signal noise and imperfections in the sample surface. When the system was set properly, the yellow and purple phases each represented about 1 percent of the total image area. During imaging, beam current and BSD calibration were checked periodically to insure stable image intensities.

Calibrating the system for videoscope settings of $-8(\mathrm{Si})$ and $-18(\mathrm{Mg})$ produced gray levels ranging from 146 to 188 for $\mathrm{Si}$ and from 27 to 97 for $\mathrm{Mg}$.

Image analysis of cement paste was carried out at magnifications of $503 \mathrm{x}$ and $1010 \mathrm{x}$. A threshold file was created for the gray level ranges of the phases within cement paste. Using the $-3(\mathrm{Si}) /-15(\mathrm{Mg})$ setting on the LAS, the gray level ranges representing the five major phases within cement paste are 1) 212 to 240 for unhydrated cement particles, 2) 159 to 211 for calcium hydroxide, 3) 107 to 158 for non-calcium hydroxide inner product, 4) 16 to 106 for calcium silicate hydrate and 5) 0 to 15 for cracks and voids. A BSE image on the IAS and the corresponding threshold image are presented in Fig. 3.

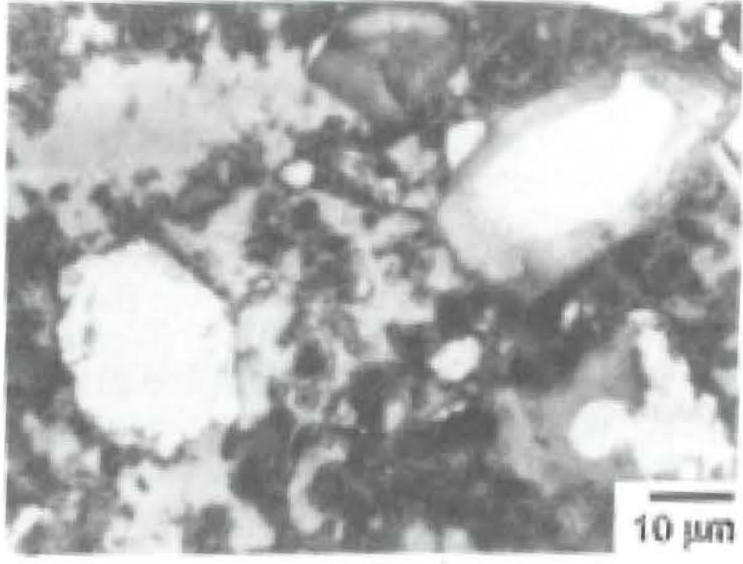

(a)

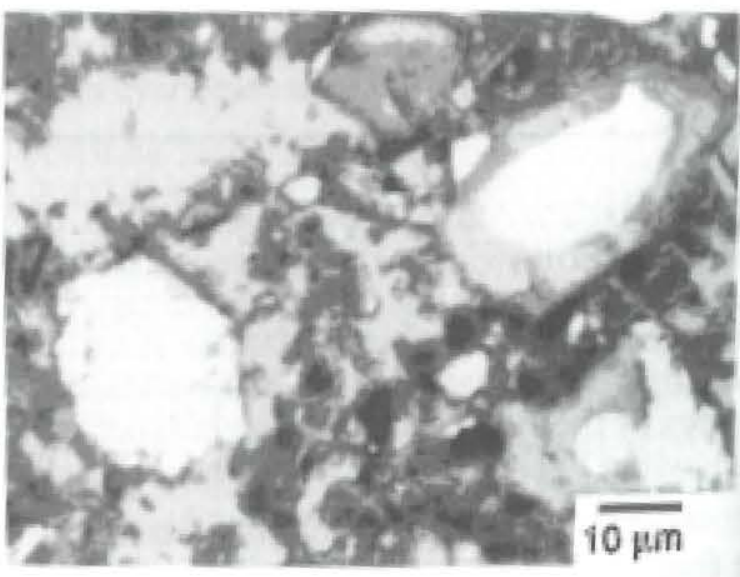

(b)

FIG. 3 (a) BSE image and (b) threshold image of cement paste, w/c $=0.4,28$ days.

Once the gray levels corresponding to the individual phases are established, the percent area for each of the phases can be determined.

\section{Results and Discussion}

\section{The Standard And Its Materials}

The BSE imaging techniques used by other researchers $(4,6-8,12)$ are valid for the qualitative analysis of cement-based materials. However, if quantitative information is required. these techniques have the disadvantage that the intensity of identical phases may change each time the SEM and the IAS are set up. These differences in signal intensity can be adjusted by the operator; however, the adjustment is both subjective and impractical for a statistical analysis for studies in which hundreds of frames are required. 
The procedures described in this paper were developed to help insure that the phases withia cement paste can be recognized consistently. Two materials with different mean atomic numbers are necessary to allow both contrast (difference in signals) and brightness (magnitude of signals) to be established. For cement paste, the mean atomic numbers for the major constituents range from 10.76 to 16.65 , as shown in Table 2 . To be most effective, a standard must possess atomic numbers that are within the range of the materials being imaged. The standard must also be chemically stable. The silicon (atomic number 14)-magnesium (atomic number 12) standard meets both requirements.

\section{Uniformity of Grey Levels}

Scrivener (12) reported that the unhydrated materials and calcium hydroxide within cement paste have fairly uniform gray levels, explained by two sharp peaks in a histogram of the gray level distribution. During the current study, it was observed that both unhydrated materials and calcium hydroxide have fairly wide ranges of gray level, as described at the end of the Experimental Work section. By using a histogram of the gray level distribution obtained in this study, three peaks, representing unhydrated material, calcium hydroxide and calcium silicate hydrate were obtained (Fig. 4a). However, the peaks for the unhydrated particles and calcium hydroxide shown in Fig. 4 are broader than those obtained by Scrivener (12). In comparison, the histogram in Fig. $4 \mathrm{~b}$ was obtained with the LAS contrast reduced by 25 percent. The peaks become taller and sharper as the contrast on the IAS is reduced. This experiment shows that a change in the contrast settings on the IAS can change the appearance of the histogram because lower contrast compresses the gray level range on the LAS so that the peaks look sharper, suggesting a uniform gray level for specific phases. However, an image with reduced contrast provides reduced efficiency for phase identification and quantitative analysis.

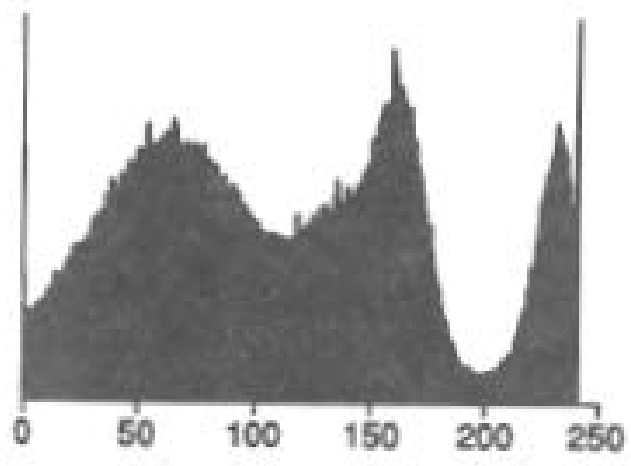

(a) Full contrast

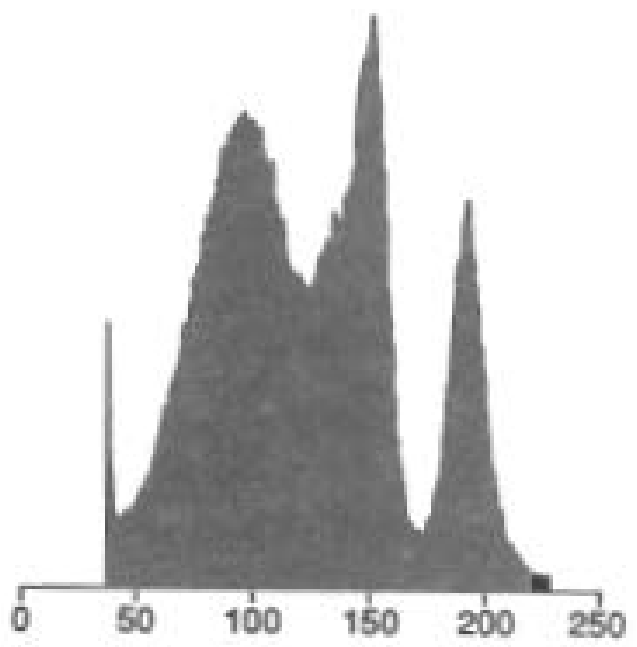

(b) $75 \%$ of full contrast

FIG. 4 Gray level histograms.

\section{Phases of Inner Product and Calcium Silicate Hydrate}

Inner product is the hydration product that forms within the original boundary of the cement gnin. If unhydrated materials are considered as a single phase, inner product is the third brightest phase in a BSE image. Typically, inner product is observed adjacent to regions of unhydrated cement (Figs. 1 and 3), but may appear independently if the specimen surface is just above or below a cement particle.

A number of investigations have found that inner product always forms around $C_{3} S(1,19$,

20). This is also observed in this study (13).

Since inner product results from the hydration of $\mathrm{C}_{3} \mathrm{~S}$, both $\mathrm{CH}$ and $\mathrm{CSH}$ should be visible vitin this region, and BSE images from the IAS give evidence of both materials within inner Fodict. When the images are transferred from the SEM to the IAS, the system distinguishes the thases based only upon intensity (gray level), which, in turn, depends on mean atomic number. 
In this study, a significant portion of inner product has the same signal intensity as massive regions of calcium hydroxide, as shown in Fig. 3.

The BSE intensity of the CSH within inner product is higher than the intensity of the CSH that forms outside of the original boundary of the cement grain. This allows these two hydration products to be distinguished from each other. This also suggests that the CSH within inner product may have a denser structure.

The fact that inner product is formed around the particles of $\mathrm{C}_{3} \mathrm{~S}$, but not $\mathrm{C}_{2} \mathrm{~S}$, is illustrated in Fig. 3. The figure, shows two prominent unhydrated cement grains, one of which is surrounded by inner product and one of which is not. X-ray analysis showed that both grains consisted of calcium silicates. The grains without inner product appear slightly darker than the grains with inner product, as would be predicted based on the slightly lower backscattering coefficient of $\mathrm{C}_{2} \mathrm{~S}$ (Table 2).

\section{Number of Frames Required for Image Analysis}

Data on phases within hydrated cement is obtained at locations across a specimen. A minimum number of frames is required to provide a level of statistical confidence in the results. The number of frames, $\mathbf{n}$, required for a 95 percent degree of confidence that the area percent of a phase is within $\delta$ of the true average can be approximated as

$$
\mathbf{n}=\left(\frac{1.96 \times \mathrm{s}}{\delta}\right)^{2}
$$

in which $\mathbf{n}$ is the estimated sample size necessary for the desired precision and confidence, $\mathbf{s}$ is the preliminary estimate of the population standard deviation of the area percent for a phase, and $\delta$ is the acceptable error or half of the maximum acceptable confidence interval (21). The sample size, $\mathbf{n}$, increases as the allowable error, $\delta$, decreases, and as the variability in the population, as denoted by $\mathbf{s}$, increases. Eq. 8 is precisely correct only for normal distributions having a known standard derivation, $\sigma$. A more accurate and more conservative estimate of $\mathbf{n}$ can be obtained if 1.96 in Eq. 8 is replaced by $t_{n-1}$, the critical value from the $t$-distribution with $n-1$ degrees of freedom, where $\mathrm{n}$ is the sample size used to estimate $\mathrm{s}$, not the number of frames calculated in Eq. 8. For large sample sizes, $t_{n-1}$ approaches 1.96. For 30 frames, Eq. 8 is within 8 percent of $n$ calculated using the $t$-distribution. The accuracy improves to 4 percent with 60 frames, which is satisfactory for most calculations.

To obtain data from the test sample and determine the number of frames required, 230 frames and 80 frames, respectively, were analyzed at magnifications of $1010 \mathrm{x}$ and $503 \mathrm{x}$. Table 3 provides the results of the analysis, including percent area, minimum number of frames, $\mathbf{n}$, and the corresponding total sampling area required for cach phase at each magnification to provide a 95 percent degree of confidence that the results are within 10 percent of the true values. The material identified as inner product, represents the non-calcium hydroxide portion of the phase. $\mathrm{CH}$ from all regions is identified as a single phase.

Table 3 shows that the number of frames required decreases as the magnification decreases, but that the total sampling area increases as the magnification decreases. For example, at a magnification of $1010 \mathrm{x}$, a minimum of 153 frames is required for unhydrated cement particles, with a corresponding area of $9.11 \times 10^{6} \mu \mathrm{m}^{2}$. At a magnification of $503 \mathrm{x}$, the same phase requires only 64 frames but a greater corresponding area, $1.54 \times 10^{7} \mu \mathrm{m}^{2}$. This phenomenon (increased area with decreased magnification) is known as the "scale effect" and has been observed in other image analysis applications (22).

One reason for this phenomenon is related to the resolution of the IAS. The size of a pixel is a fixed portion of the viewing area. Thus, the area represented by a single pixel varies with the magnification. At a magnification of $500 \mathrm{x}$, a pixel represents four times the area of a pixel at $1000 \mathrm{x}$. The effect is to reduce the clarity of boundaries between phases as the magnification is decreased. Since the gray level of a pixel on a boundary will be the average of the gray levels from two phases, the IAS must assign that pixel based on an intensity that does not accurately represt either phase. Depending on the grey level ranges assigned to individual phases, the pixel may 
assigned to one of the adjacent phases or even to a third phase. Therefore, as magnification is lowered, the system tends to lose detailed information about features. This leads to an increase in the scatter in the percent area for a phase.

TABLE 3

Area percent analysis of 28-day old cement paste

including the number of frames required for 95 percent confidente that the area percent is within 10 percent of the measured value

\begin{tabular}{lcccc}
\hline Phases & $\begin{array}{c}\text { Percent } \\
\text { Area } \\
\%\end{array}$ & $\begin{array}{c}\text { Standard } \\
\text { Deviation } \\
\mathbf{s}\end{array}$ & $\begin{array}{c}\text { Number of } \\
\text { Frames Required } \\
\mathbf{n}\end{array}$ & $\begin{array}{c}\text { Corresponding } \\
\text { Area Required } \\
\left(\mu \mathrm{m}^{2}\right)\end{array}$ \\
\hline \multicolumn{5}{c}{ Magnification $-1010 \mathrm{x}$} \\
UH & 10.123 & 6.385 & 153 & $9.11 \times 10^{6}$ \\
CH & 15.637 & 3.899 & 23 & $1.36 \times 10^{\circ}$ \\
IP* & 22.830 & 3.087 & 7 & $4.16 \times 10^{*}$ \\
CSH & 44.391 & 5.840 & 7 & $4.16 \times 10^{*}$ \\
Cracks & 6.979 & 3.006 & 71 & $4.23 \times 10^{6}$ \\
\& Voids & & & & \\
\hline
\end{tabular}

Magnification - $503 \mathrm{x}$

\begin{tabular}{lrccc}
\hline UH & 10.400 & 4.24 & 64 & $1.54 \times 10^{7}$ \\
CH & 15.577 & 3.11 & 16 & $3.84 \times 10^{6}$ \\
IP* & 22.011 & 1.98 & 4 & $9.60 \times 105$ \\
CSH & 44.153 & 4.21 & 4 & $9.60 \times 10^{5}$ \\
Cracks & 7.860 & 3.12 & 61 & $1.46 \times 10^{7}$ \\
\& Voids & & & \\
"Non-CH portion of IP & & & \\
\hline
\end{tabular}

From Table 3 it can also be noted that to achieve the desired degree of confidence that the area pereent of each phase is within 10 percent of the measured value, the number of frames required for each phase is different. At a magnification of $1010 \mathrm{x}, 153$ frames are required for unhydrated cement particles, compared to 23 frames for calcium hydroxide, 7 frames for inner product and calcium silicate hydrate, and 71 frames for cracks and voids. Note that unhydrated cement particles require the greatest number of frames, while calcium silicate hydrate and inner product require the least. The same tendency is apparent for the results obtained at $503 \mathrm{x}$.

\section{Conclusions} Paper.

The following conclusions are based on the experimental work and analyses presented in this

1. A silicon-magnesium standard can be used to establish an objective method for setting both a scanaing electron microscope and an image analysis system for quantitative backscattered tlactron analysis of hydrated cement paste.

2. Backscattered electron imaging of polished surfaces of epoxy impregnated cement paste

a be used to distinguish unhydrated particles, calcium hydroxide, the non-calcium bydroxide

prion of inner product, calcium silicate hydrate, and cracks and voids.

3. For image analysis, the number of frames required for a selected degree of confidence creases as the magnification decreases, while the total area required increases as the magnifica- 
tion decreases. This is due to the scale effect.

4. To achieve a desired degree of confidence in image analysis for the phases of hydrated cement paste, unhydrated cement particles require the greatest number of frames, while inner product and calcium silicate hydrate require the least number of frames.

\section{Acknowledgements}

This report is based on research performed by Hong Zhao in partial fulfiliment of the requirements for the MSCE degree from the University of Kansas. This research was supported by the Air Force Office of Scientific Research under Grant AFOSR-89-0296.

\section{References}

1. Williamson, R. B., Progr, in Mat. Sci., 15189 (1972).

2. Diamond, S., Proc., Conf, on Hydraulic Cem. Pastes: Their Structure and Properties, Cem. Concr. Assn., Univ. of Sheffield, England, 2 (1976).

3. Dalgleish, B. J. and Pratt, P. L., J. Mats. Sci., 172199 (1982).

4. Scrivener, K. L and Pratt, P. L., Proc., 6th Int. Conf. on Cem. Microscopy, Albuquerque, 145 (1984).

5. Attiogbe, E. K. and Darwin, D., ACl Mat. J.84 491, (1987).

6. Scrivener, K. L., Baldie, K. D., Halse, Y. and Pratt, P. L., Mat. Res. Soc. Symp. Proc., 42 40 (1985).

7. Scrivener, K. L. and Gartner, E. M., Mat, Res. Soc. Symp. Proc., 11477 (1988).

8. Kjellsen, K., Detwiler, R., and Gjørv, O., Cem. Concr. Res., 20308 (1990).

9. Struble, L. and Byrd, E., Progress Report, NBSIR 87-3504 (1986).

10. Struble, L. and Stutzman, P., Progress Report, NBSIR 88-3702 (1988).

11. Kjellsen, K., Detwiler, R., and Gjørv, O., Cem. Concr. Res. 21388 (1991).

12. Scrivener, K. L., Mat. Res. Soc. Symp. Proc., 137129 (1989).

13. Zhao, H. and Darwin, D., SM Report No. 24, Univ. of Kansas Center for Research, Lawrence, Kansas (1990).

14. Kanaya, K. and Okayama, S., J. Physics. D: Applied Physics, 543 (1972).

15. Heinrich, K. F. J., Proc, 4th Int. Cong. on X-Ray Optics and Microanalysis, Hermann, Paris, 1509 (1966).

16. Goldstein, I., Newbury, D., Echlin, P., Joy, D., Fiori, C.and Lifshin, E., Scanning Electron Microscopy and X-Ray Microanalysis, Plenum Press, New York and London (1981).

17. Oatley, C. W., Nixon, W. C., and Pease, R. F. W.. Advances in Electronics and Electron Physics, Academic, New York, 181 (1965).

18. Newbury, D. E., Joy, D. C., Echlin, P., Fiom, C. E., and Goldstein, J. I., Advanced Scanning Electron Microscopy and X-Ray Micronalysis, Plenum Press, New York, and London (1986).

19. Brown, L. S, and Carlson, R. W., Proc., Amer. Soc. Test. and Mat., 36332 (1936).

20. Stucke, M. S. and Majumdar, A. J., Proc., Conf, on Hydraulic Cem. Pastes: Their Structure and Properties, Cem. Conc. Assn, Univ. of Sheffield, England, 31 (1976).

21. Ostle, B. and Malone, L. C., Statistics in Research, lowa State Univ. Press, 4th Bd. (1988).

22. Serra, J., Image Analysis and Mathematical Morphology. Academic Press, London, New York, Toronto (1982). 